from which it follows that (see [9])

$$
\lim _{a, b \rightarrow \infty}(a b)^{-1} M(a, b)=\left(\lim _{a \rightarrow \infty} a^{-1} m(a)\right)^{2} \equiv \eta^{2},
$$

which is the Palásti conjecture for the Rényi model. Similar results hold for the Solomon model.

University of California at Davis

Yours sincerely, HOWARD WEINER

Dear Editor,

\title{
A critique of Weiner's work on Palásti's conjecture
}

Weiner [9] presented an argument purporting to prove Palásti's conjecture for Rényi and Solomon random parking schemes. This argument was criticized in letters to the editor by Tory and Pickard [8], Tanemura [7], and Hori [4], and Weiner responded [10]. Weiner [11] has now offered an alternative argument. In this letter, we point out various instances of the fundamental error which Weiner makes. This error invalidates not only his specific results but also his entire approach. Indeed, it is now apparent that very little of Weiner's work on Palásti's conjecture can withstand close scrutiny.

The notion of exchangeability is germane. A collection of random variables, indexed by $I$, is exchangeable if the likelihood is invariant under permutations of $I$. By exchangeability for sequential random packing schemes we shall mean that all realizations leading to the same final configuration have the same likelihood; i.e. the invariance is under permutations of the order in which the particles are packed. Tory and Pickard have pointed out the rather obvious fact that, in this sense, the Rényi model is not exchangeable (see Figure 2 and the related discussion in Tory and Pickard [8]). Clearly, neither is the Solomon model.

Weiner's fundamental error. To compare averages for different parking procedures, Weiner repeatedly (in both [9] and [11]) argues solely on the basis of related individual final configurations. That is, he consistently ignores the role in determining such averages played by the relative frequencies with which these configurations occur. These frequencies are different for the different models, so arguments relating averages via realizations must also involve the frequencies. Weiner's arguments do not. Furthermore, since the parking procedures are non-exchangeable, the relative frequencies are far too complicated to permit such comparisons. Consequently, Professor Weiner's work cannot be 'corrected'

Received 4 January 1980. 
by mere fiddling with lemmas and/or proofs. Rather, his entire approach is doomed from the outset, and must be abandoned.

More formally, consider two probability spaces, $(\Omega, \mathscr{F}, P)$ and $\left(\Omega^{*}, \mathscr{F}^{*}, P^{*}\right)$, and two random variables $X: \Omega \rightarrow \mathbf{R}, X^{*}: \Omega^{*} \rightarrow \mathbf{R}$. Let $\phi$ be a measurable map from $\Omega$ onto $\Omega^{*}$. Now suppose that some linear relationship (equality or inequality) holds on $\Omega$ between $X$ and $X^{*} \circ \phi$. Does it necessarily hold between $\mathscr{E}_{P} X$ and $\mathscr{E}_{P} \cdot X^{*}$ as well? Of course not! - it depends on how $P^{*}$ and the measure $P_{\phi}$ induced on $\left(\Omega^{*}, \mathscr{F}^{*}\right)$ by $\phi$ and $(\Omega, \mathscr{F}, P)$ are related.

Obviously, any linear relationship between $X$ and $X^{*} \circ \phi$ will be inherited by their expectations (with respect to $P$ ) and consequently by $\mathscr{C}_{P} X$ and $\mathscr{E}_{P_{\phi}} X^{*}$ as well since

$$
\mathscr{C}_{P} X^{*} \circ \phi=\int_{\Omega} X^{*} \circ \phi(\omega) d P(\omega)=\int_{\Omega^{*}} X^{*}\left(\omega^{*}\right) d P_{\phi}\left(\omega^{*}\right)=\mathscr{C}_{P_{\phi}} X^{*}
$$

However, Weiner claims such relationships for $\mathscr{E}_{\mathrm{P}} X$ and

$$
\mathscr{C}_{P} \cdot X^{*}=\int_{\Omega^{*}} X^{*}\left(\omega^{*}\right) d P^{*}(\omega)
$$

in contexts where the measures $P^{*}$ and $P_{\phi}$ are clearly different. Therefore, $\mathscr{C}_{P} X$ and $\mathscr{E}_{P} \cdot X^{*}$ cannot be compared via the correspondence $\phi$ without also comparing the $P^{*}$ and $P_{\phi}$ likelihoods.

Remark. It is perhaps relevant to note that in some of Weiner's arguments $P^{*}$ and $P_{\phi}$ would be identical if only Rényi parking were exchangeable.

\section{Examples of the fundamental error}

Example 1. Lemma 2 of [9]. Weiner appeals to this in both new [11] and old [9] versions of his Lemma 3. Lemma 2 states that the first row in a 2-D Rényi model is essentially a 1-D Rényi model. Tory and Pickard [8] have pointed out Weiner's failure to prove this. Each final configuration in the first row of the 2-D model $(\Omega, \mathscr{F}, P)$ corresponds (thereby defining $\phi)$ to a final configuration for a 1-D model $\left(\Omega^{*}, \mathscr{F}^{*}, P^{*}\right)$. However, it is not at all clear that corresponding configurations have the same relative frequency of occurrence in the two models (i.e. that $P^{*} \equiv P_{\phi}$ ). Basically, the problem is that in 2-D, cars parked near the first row but not in it affect the subsequent parking in the first row (see Figure 1 in Tory and Pickard [8]). If Rényi parking were exchangeable this problem would disappear (i.e. $P^{*}$ and $P_{\phi}$ would be identical) for then we could simply park the first row first, i.e. essentially according to a 1-D Rényi model.

In [10] Weiner simply claims that averaging over all ordered parkings compatible with a given final configuration in the first row will result in the same relative frequencies in both the 1-D and 2-D models. This is rather doubtful, but in any case Weiner must prove it, and not merely claim it as 'obvious'. 
Example 2. (1.3) of [11]. This says that on average one can park more cars in a larger lot. This must surely be true! However, Weiner has not proved it.

His 'proof' goes as follows: pack $(a+1) \times b$ with unit squares according to Rényi, contract the $x$ coordinate by $a /(a+1)$; expand cars to unit squares, and then carefully remove any overlapping cars. This results in a packing of $a \times b$.

If this procedure assigned the same likelihood to each configuration as an $a \times b$ Rényi pack, Weiner could argue as follows: The Rényi $(a+1) \times b$ packing always dominates the shrinking $a \times b$ packing, so its average dominates the shrinking one; also, the shrinking and Rényi $a \times b$ packings are identical, so the Rényi $(a+1) \times b$ packing dominates the Rényi $a \times b$ one.

For Weiner's proof to go through it will suffice to show that the average for the shrinking $a \times b$ packing equals or is larger than that for the Rényi $a \times b$ packing. However, if the $a \times b$ Rényi model gives more weight to the denser configurations, this simply will not be true. In any case, the incredibly complicated nature of the likelihoods for these models renders the entire approach hopeless.

Again, were Rényi parking exchangeable the result could be easily proved. By exchangeability, in a Rényi pack of $(a+1) \times b$, configurations on an $a \times b$ subset have the same likelihood as in an $a \times b$ Rényi model (just pack $a \times b$ first) i.e. the induced measure (on the subset) is identical to the measure requiring comparison (the $a \times b$ Rényi model).

Example 3. (1.4) and (1.5) of [11]. Weiner merely claims that (1.4) and (1.5) follow from Lemma 2 of [9] by arguments similar to that used to prove (1.3). So far Weiner has failed to prove either Lemma 2 or (1.3).

However, from Lemma 3 of [9] the intuitive sense of his arguments appears to be essentially as follows. For (1.4): the first row of $(a+1) \times b$ is packed by a 1-D Rényi model, also the remaining region is contained in the $a \times b$ subset and consequently parks fewer cars on average than an $a \times b$ Rényi model. For (1.5): the first row of $(a+2) \times b$ is packed by a 1-D Rényi model, also the remaining region contains the $a \times b$ subset and consequently parks more cars on average than an $a \times b$ Rényi model. The first parts of these arguments require Lemma 2 which is unsubstantiated. The second parts involve comparing $a \times b$ Rényi models to parking in the remaining region given the configuration of the first row. However, since Rényi parking is non-exchangeable, we cannot assume that this first row of cars was parked first, and consequently, the conditional parking procedure is not even Rényi (i.e. it is not random in the available space). Consequently, the relative frequencies become so confused that the necessary comparisons cannot be made. Notice that although Rényi parking averages seem likely to increase on expanding regions, such a result is useless here because the conditional models are not Rényi.

Again, the difficulties would all dissolve if only Rényi parking were exchange- 
able for in this case, the first row could then be parked first, the conditional model would then be Rényi, and Rényi parking averages could be easily shown to increase on expanding regions.

Example 4. (2.5), (2.6) of [9]. These are essentially the results (1.3), (1.4), and (1.5) of [11]. The original proof also depends critically on Lemma 2. Weiner claims (2.7) for all rows, apparently via Lemma 2 , and presumably by conditioning as in Example 3 above. Consequently, the criticisms above again apply.

Example 5. Theorem 1 of [11], Lemma 4 of [9]. These are essentially the same results. In [11] Weiner presents more of his reasoning. For (1.6) and (1.7) he appeals to inductions on (1.4) and (1.5) respectively, neither of which he has been able to prove. However, the basic idea behind the 'proof' fails as well.

$\mathrm{He}$ begins by parking the first car and then partitioning $a \times b$ into four rectangles by forming the unit horizontal and vertical strips containing that first car. The strips are then packed as 1-D Rényi models with the first car already parked. Finally, the four rectangles are packed according to 2-D Rényi models. Thus, we have two packing procedures for $a \times b$, the basic Rényi model, and this partitioned one. The LHS of (1.6) is the average number of cars parked for the Rényi model while the RHS is the corresponding quantity for the partitioned model. Weiner claims, but certainly does not prove (despite the reference to an induction on (1.4)), that the partitioned model will park more cars on average.

The intuitive sense of the argument would seem to be as follows: compared to a Rényi pack of $a \times b$, the strips are more densely covered while the rectangles ought to be about right. In the same vein one might argue that the extra boundaries result in less dense packing in the rectangles so that the net effect is unclear. However, all such arguments are worthless as they do not incorporate the relative frequencies with which the configurations arise under the two different models.

Surprisingly, exchangeability for Rényi parking would actually reverse inequality (1.6). Indeed, if the rectangles were parked first it is apparent that one could do better than 1-D Rényi packs in the strips because occasionally two cars would slip in.

A similar argument is claimed for (1.7). Presumably, this time the strips are three car widths and the argument is that the strips are relatively underpacked. The same criticism applies and under exchangeability, since (1.6) reverses, (1.7) certainly holds.

Comments. (1.10) of [11], (2.13) of [9]. These are essentially the same results. Moreover, they imply Palásti's conjecture. However, Weiner's 'proof' relies on Theorem 1 of [11] or equivalently Lemma 4 of [9] which he has been unable to prove. 
The right-hand inequality, (1.10-rh), does follow by induction from (1.6) on replacing $M$ by $R$ in the integrand of (1.6). However, (1.7) merely implies that

$$
M(a+2, b+2)-R(a, b) \geqq \frac{4}{(a-1)(b-1)} \int_{0}^{a-1} \int_{0}^{b-1}(M(\varepsilon, \eta)-R(\varepsilon, \eta)) d \varepsilon d \eta
$$

where the quantity on the right is negative by $(1.10-r h)$. Consequently, even given Theorem 1, the left-hand inequality, (1.10-lh), would still be unproved.

Conclusions. The only parts of Weiner's edifice which remain intact are Lemma 1 and the following pair of inferences: (1.6) implies (1.10-rh), and (1.10) implies Palásti's conjecture. This is not to say that the other results are necessarily false, but rather that Weiner has failed to prove them. Indeed, (1.3) of [11] is assuredly true. Now, (1.10-rh) obviously implies that the limiting parking density is not greater than Palásti's conjecture of $\eta^{2}$. On the other hand, simulations indicate otherwise (cf. Akeda and Hori [1], Blaisdell and Solomon [2], Jodrey and Tory [5]). Therefore, it is most important to determine the status of (1.6) and (1.10-rh) of [11].

Recall that the LHS of (1.10) is the average number (say $N_{1}$ ) of cars parked in an $a \times b$ Rényi model, while the RHS is this average (say $N_{2}$ ) for the partitioned model discussed in Example 5 above. The obvious way to compare these averages, which are $O(a b)$, is to consider boundary effects. Assuming Palásti's conjecture actually holds, the correction terms (Dvoretzky and Robbins [3]) then imply that the difference between these averages (i.e. $\left.N_{1}-N_{2}\right)$ is $3(1-\eta)^{2}+$ $o(1)$. That is, even if the conjecture is true, Weiner's critical inequalities (1.6) and (1.10-rh) cannot hold - indeed, they must be reversed. Finally if, as simulations suggest, the limiting parking density is actually greater than $\eta^{2}$, these critical inequalities must again be reversed.

Harvard University

Yours sincerely, David K. Pickard

Mount Allison University

E. M. TORY

Dear Editor,

Some comments on the letters by $H$. J. Weiner

In this letter, I should like to comment on Weiner's reply [10] to my first letter [7] and to the letters of other authors, and on Weiner's recent alternative argument [11] for his paper [9]. The conclusion of [11] does not differ from that of [9]. I will discuss in this note the following two points:

Received 12 December 1979. 